\title{
Sampled-data fuzzy controller for continuous nonlinear systems
}

\author{
H.K. Lam and W.K. Ling \\ Department of Electronic Engineering, Division of Engineering, The King's College \\ London, Strand, London, WC2R 2LS, United Kingdom
}

\begin{abstract}
This paper presents the sampled-data fuzzy control of nonlinear systems. The consequents of the fuzzy controller rules are linear sampled-data sub-controllers. As a result, the fuzzy controller is a weighted sum of some linear sampled-data sub-controllers which can be implemented by a microcontroller or a digital computer to lower the implementation cost. Consequently, a hybrid fuzzy controller consisting of continuoustime grades of memberships and discrete-time sub-controller is resulted. The system stability of the fuzzy control system is investigated. The proposed fuzzy controller exhibits a favourable property to alleviate the conservativeness of the stability analysis. Due to the sampling activity introduces discontinuity to complicate the system dynamics, the stability analysis is made difficult. Lyapunov-based approach is employed to investigate the system stability. Furthermore, LMI (linear matrix inequality)-based performance conditions are derived to guarantee the system performance of the fuzzy control system. An application example is given to illustrate the merits of the proposed approach.
\end{abstract}

\section{INTRODUCTION}


Fuzzy control approach offers a powerful and systematical control methodology to handle nonlinear systems. Due to the superior approximation and reasoning abilities of the fuzzy controller, fuzzy control approach has been applied in different applications. One of the most common issues of the fuzzy control systems is the system stability which has drawn the attention of researchers for many years. In the early stage, the fuzzy controllers have been designed heuristically. However, the fuzzy controllers designed based on the heuristic techniques come with no guarantee on the system stability. With the extensive efforts of the researchers working on the fuzzy control discipline, fruitful stability analysis results have been obtained to aid the design of stable fuzzy controllers.

The most popular method to study the stability of the fuzzy control systems is the Lyapunov-based approach. In [1]-[2], a T-S fuzzy model was employed to represent the system dynamics of the nonlinear system. The T-S fuzzy model represents the nonlinear system as a weighted sum of some linear sub-systems. This particular structure offers a general framework to represent the nonlinear system which is favourable for system analysis. A fuzzy controller [3]-[4] was proposed to handle the nonlinear system represented the fuzzy model. The fuzzy control system is guaranteed to be asymptotically stable if there exists a solution to a set of linear matrix inequalities (LMIs) [5] which can be solved efficiently and numerically using some convex programming techniques. In [4], a parallel distributed compensation (PDC) design approach was proposed to design the feedback gains of the fuzzy controllers. It was proposed in [4], [6] that the fuzzy controller shares the same premises as those of the fuzzy model. Under such a design criterion, it was reported in [6] that relaxed stability conditions can be obtained. Further relaxed stability results were reported in [7]-[11]. 
In most of the work on the stability analysis of fuzzy control systems, both continuous-time and discrete-time fuzzy control system have been studied. However, fuzzy controllers with sampled-data control rules are seldom concerned. The sampleddata control rules can be easily implemented by some microcontrollers or digital computers to lower the implementation cost and time. However, the system dynamics of the closed-loop system will become more complex due to the sampling activity introducing discontinuity to the system which increases the analysis difficulty. As a result, the stability analysis approach proposed in pure continuous-time or discrete-time fuzzy controller system cannot be applied. In [12], the sampled-data fuzzy control system was represented by an equivalent jump system. In this approach, the system is separated to continuous and dis-continuous parts. The system stability is implied by the stabilities of both parts. In [13], the system stability of a linear sampled-data control system was analyzed. By representing the sampling time as a time-varying delay, stability conditions were derived using Lyapunov-based approach. However when nonlinear systems are considered, the system analysis will become more complicated. In [14], we have extended the approach for linear system in [13] to time-delay sampled-data fuzzy control systems. As the membership functions depending on the sampled system states, which leads to the favourable property relaxing the stability conditions, vanishes, a conservative stability analysis result were obtained. Furthermore, it leads to a more number of stability conditions.

In this paper, fuzzy controller with sampled-data control rules is proposed to handle the nonlinear plant represented by a fuzzy model. In the proposed fuzzy controller, it shares the same membership functions as those of the fuzzy model to relax 
the stability conditions. In the consequent part, the fuzzy controller employs sampleddata linear sub-controllers. The sampled-data linear sub-controller is formed by a sampler with sampling period $h$, a discrete-time linear controller and a zero-order-hold $(\mathrm{ZOH})$ unit. In this paper, the system stability of this kind of fuzzy control system is investigated using Lyapunov-based approach. LMI-stability conditions are derived to guarantee the system stability. In order to guarantee the system performance, LMI-based performance conditions are derived as well. The stability and performance conditions will serve as a mathematical tool to aid the design of a stable and well-performed fuzzy controller for nonlinear system.

This paper is organized as follows. In section II, the fuzzy model and the fuzzy controller with sampled-data control rules are presented. In section III, the LMI-based stability and performance conditions of the fuzzy control systems are derived. In section IV, an application example is presented to illustrate the design procedure and the merits of the proposed approach. A conclusion is drawn in section V.

\section{FuZzy Model AND FuZzy Controller With SAMPLED-DAta CONTROL Rules}

A fuzzy control system, formed by a nonlinear plant represented by a fuzzy model and a fuzzy controller with sampled-data control rules, is considered.

\section{A. Fuzzy Model}

Let $p$ be the number of fuzzy rules describing the nonlinear plant. The $i$-th rule is of the following format,

Rule $i$ : IF $f_{1}(\mathbf{x}(t))$ is $\mathbf{M}_{1}^{i}$ AND ... AND $f_{\psi}(\mathbf{x}(t))$ is $\mathbf{M}_{\psi}^{i}$ 


$$
\text { THEN } \dot{\mathbf{x}}(t)=\mathbf{A}_{i} \mathbf{x}(t)+\mathbf{B}_{i} \mathbf{u}(t)
$$

where $\mathrm{M}_{\alpha}^{i}$ is a fuzzy term of rule $i$ corresponding to the function $f_{\alpha}(\mathbf{x}(t)), \alpha=1,2, \ldots$, $\Psi, i=1,2, \ldots, p, \Psi$ is a positive integer; $\mathbf{A}_{i} \in \mathfrak{R}^{n \times n}$ and $\mathbf{B}_{i} \in \mathfrak{R}^{n \times m}$ are known constant system and input matrices respectively; $\mathbf{x}(t) \in \mathfrak{R}^{n \times 1}$ is the system state vector and $\mathbf{u}(t) \in \mathfrak{R}^{m \times 1}$ is the input vector. The system dynamics are described by,

$\dot{\mathbf{x}}(t)=\sum_{i=1}^{p} w_{i}(\mathbf{x}(t))\left(\mathbf{A}_{i} \mathbf{x}(t)+\mathbf{B}_{i} \mathbf{u}(t)\right)$

where,

$$
\begin{aligned}
& \sum_{i=1}^{p} w_{i}(\mathbf{x}(t))=1, w_{i}(\mathbf{x}(t)) \in\left[\begin{array}{ll}
0 & 1
\end{array}\right] \text { for all } i \\
& w_{i}(\mathbf{x}(t))=\frac{\mu_{\mathrm{M}_{1}^{i}}\left(x_{1}(t)\right) \times \mu_{\mathrm{M}_{2}^{i}}\left(x_{2}(t)\right) \times \cdots \times \mu_{\mathrm{M}_{n}^{i}}\left(x_{n}(t)\right)}{\sum_{k=1}^{p}\left(\mu_{\mathrm{M}_{1}^{k}}\left(x_{1}(t)\right) \times \mu_{\mathrm{M}_{2}^{k}}\left(x_{2}(t)\right) \times \cdots \times \mu_{\mathrm{M}_{n}^{k}}\left(x_{n}(t)\right)\right)}
\end{aligned}
$$

is a nonlinear function of $\mathbf{x}(t)$ and $\mu_{\mathrm{M}_{\alpha}^{i}}\left(x_{\alpha}(t)\right)$ is the grade of membership corresponding to the fuzzy terms $\mathrm{M}_{\alpha}^{i}$.

\section{B. Fuzzy Controller with Sampled-Data Control Rules}

A p-rule fuzzy controller is designed based on the fuzzy model of the nonlinear plant. The $j$-th rule of the fuzzy controller is of the following format:

Rule $j$ : IF $f_{1}(\mathbf{x}(t))$ is $\mathrm{M}_{1}^{j}$ AND ... AND $f_{\Psi}(\mathbf{x}(t))$ is $\mathrm{M}_{\Psi}^{j}$

$$
\operatorname{THEN} \mathbf{u}(t)=\mathbf{G}_{j} \mathbf{x}\left(t_{\gamma}\right), t_{\gamma}<t \leq t_{\gamma+1}
$$

where $\mathbf{G}_{j} \in \mathfrak{R}^{m \times n}$ is the feedback gain of rule $j$ to be designed. $t_{\gamma}=\gamma h, \gamma=0,1,2, \ldots, \infty$; denotes a time instant; $h=t_{\gamma+1}-t_{\gamma}$ denotes the constant sampling period. It can be seen 
from (5) that $\mathbf{u}(t)=\mathbf{u}\left(t_{\gamma}\right)$ of each individual rule holds constant vector value for $t_{\gamma}<t \leq$ $t_{\gamma+1}$. The inferred output of the fuzzy controller is given by,

$\mathbf{u}(t)=\sum_{j=1}^{p} w_{j}(\mathbf{x}(t)) \mathbf{G}_{j} \mathbf{x}\left(t_{\gamma}\right), t_{\gamma}<t \leq t_{\gamma+1}$

Denote $\tau(t)=t-t_{\gamma} \leq h$ for $t_{\gamma}<t \leq t_{\gamma+1}$ and from (6), we have,

$$
\begin{aligned}
\mathbf{u}(t) & =\sum_{j=1}^{p} w_{j}(\mathbf{x}(t)) \mathbf{G}_{j} \mathbf{x}\left(t-\left(t-t_{\gamma}\right)\right) \\
& =\sum_{j=1}^{p} w_{j}(\mathbf{x}(t)) \mathbf{G}_{j} \mathbf{x}(t-\tau(t))
\end{aligned}
$$

\section{Stability Analysis And Performance Design}

In this section, the system stability of the fuzzy control system formed by (2) and (7) is investigated based on the Lyapunov-based approach. Stability conditions are derived to guarantee the system stability. Based on the stability conditions, the feedback gains $\mathbf{G}_{j}, j=1,2, \ldots, p$, can be determined to achieve the system stability. LMI-based performance conditions will be derived to serve as design constraints for the feedback gains to achieve the system performance.

\section{A. Stability Analysis}

The system stability of the fuzzy control system is investigated. In the following analysis, $w_{i}(\mathbf{x}(t))$ is denoted by $w_{i}$ for short. Furthermore, the equality of $\sum_{i=1}^{p} w_{i}=$ $\sum_{i=1}^{p} \sum_{j=1}^{p} w_{i} w_{j}=1$ is used. The control action of (7) can be written as follows. 


$$
\mathbf{u}(t)=\sum_{j=1}^{p} w_{j} \mathbf{G}_{j} \mathbf{x}(t)-\sum_{j=1}^{p} w_{j} \mathbf{G}_{j} \int_{t-\tau(t)}^{t} \dot{\mathbf{x}}(\varphi) d \varphi
$$

From (8), we have the following equality which is used in the following stability analysis.

$$
\sum_{j=1}^{p} w_{j}\left[\begin{array}{cc}
\mathbf{0} & \mathbf{0} \\
\mathbf{G}_{j} & -\mathbf{I}
\end{array}\right]\left[\begin{array}{l}
\mathbf{x}(t) \\
\mathbf{u}(t)
\end{array}\right]-\sum_{j=1}^{p} w_{j}\left[\begin{array}{c}
\mathbf{0} \\
\mathbf{G}_{j}
\end{array}\right] \int_{t-\tau(t)}^{t} \dot{\mathbf{x}}(\varphi) d \varphi=\left[\begin{array}{l}
\mathbf{0} \\
\mathbf{0}
\end{array}\right]
$$

To investigate the system stability of the fuzzy control system, the following Lyapunov function candidate is considered,

$V(t)=V_{1}(t)+V_{2}(t)$

where

$V_{1}(t)=\mathbf{x}(t)^{\mathrm{T}} \mathbf{P}_{1} \mathbf{x}(t)$

$V_{2}(t)=\int_{-h}^{0} \int_{t+\sigma}^{t} \dot{\mathbf{x}}(\varphi)^{\mathrm{T}} \mathbf{R} \dot{\mathbf{x}}(\varphi) d \varphi d \sigma$

and $\mathbf{P}_{1}=\mathbf{P}_{1}^{\mathrm{T}} \in \mathfrak{R}^{n \times n}>0$ and $\mathbf{R}=\mathbf{R}^{\mathrm{T}} \in \mathfrak{R}^{n \times n}>0$. It will be shown that $\dot{V}(t) \leq 0$ (equality holds when $\mathbf{x}(t)=\mathbf{0}$ and $\mathbf{u}(t)=\mathbf{0})$ which implies the asymptotic stability of the fuzzy control system. From (2), (8), (9), (11),

$$
\begin{aligned}
\dot{V}_{1}(t) & =\mathbf{x}(t)^{\mathrm{T}} \mathbf{P}_{1} \dot{\mathbf{x}}(t)+\dot{\mathbf{x}}(t)^{\mathrm{T}} \mathbf{P}_{1} \mathbf{x}(t) \\
& =\sum_{i=1}^{p} w_{i}\left[\begin{array}{l}
\mathbf{x}(t) \\
\mathbf{u}(t)
\end{array}\right]^{\mathrm{T}}\left(\left[\begin{array}{cc}
\mathbf{P}_{1} & \mathbf{0} \\
\mathbf{0} & \mathbf{0}
\end{array}\right]\left[\begin{array}{cc}
\mathbf{A}_{i} & \mathbf{B}_{i} \\
\mathbf{0} & \mathbf{0}
\end{array}\right]+\left[\begin{array}{cc}
\mathbf{A}_{i} & \mathbf{B}_{i} \\
\mathbf{0} & \mathbf{0}
\end{array}\right]^{\mathrm{T}}\left[\begin{array}{cc}
\mathbf{P}_{1} & \mathbf{0} \\
\mathbf{0} & \mathbf{0}
\end{array}\right]\right)\left[\begin{array}{l}
\mathbf{x}(t) \\
\mathbf{u}(t)
\end{array}\right]
\end{aligned}
$$




$$
\begin{aligned}
& =\sum_{i=1}^{p} w_{i}\left[\begin{array}{l}
\mathbf{x}(t) \\
\mathbf{u}(t)
\end{array}\right]^{\mathrm{T}}\left(\left[\begin{array}{cc}
\mathbf{P}_{1} & \mathbf{0} \\
\mathbf{P}_{2} & \mathbf{P}_{3}
\end{array}\right]^{\mathrm{T}}\left[\begin{array}{cc}
\mathbf{A}_{i} & \mathbf{B}_{i} \\
\mathbf{0} & \mathbf{0}
\end{array}\right]+\left[\begin{array}{cc}
\mathbf{A}_{i} & \mathbf{B}_{i} \\
\mathbf{0} & \mathbf{0}
\end{array}\right]^{\mathrm{T}}\left[\begin{array}{cc}
\mathbf{P}_{1} & \mathbf{0} \\
\mathbf{P}_{2} & \mathbf{P}_{3}
\end{array}\right]\right)\left[\begin{array}{l}
\mathbf{x}(t) \\
\mathbf{u}(t)
\end{array}\right] \\
& +\sum_{i=1}^{p} w_{i}\left[\begin{array}{l}
\mathbf{x}(t) \\
\mathbf{u}(t)
\end{array}\right]^{\mathrm{T}}\left(\left[\begin{array}{cc}
\mathbf{P}_{1} & \mathbf{0} \\
\mathbf{P}_{2} & \mathbf{P}_{3}
\end{array}\right]^{\mathrm{T}}\left[\begin{array}{cc}
\mathbf{0} & \mathbf{0} \\
\mathbf{G}_{i} & -\mathbf{I}
\end{array}\right]+\left[\begin{array}{cc}
\mathbf{0} & \mathbf{0} \\
\mathbf{G}_{i} & -\mathbf{I}
\end{array}\right]^{\mathrm{T}}\left[\begin{array}{cc}
\mathbf{P}_{1} & \mathbf{0} \\
\mathbf{P}_{2} & \mathbf{P}_{3}
\end{array}\right]\right)\left[\begin{array}{l}
\mathbf{x}(t) \\
\mathbf{u}(t)
\end{array}\right] \\
& -2 \sum_{i=1}^{p} w_{i}\left[\begin{array}{c}
\mathbf{x}(t) \\
\mathbf{u}(t)
\end{array}\right]^{\mathrm{T}}\left[\begin{array}{cc}
\mathbf{P}_{1} & \mathbf{0} \\
\mathbf{P}_{2} & \mathbf{P}_{3}
\end{array}\right]^{\mathrm{T}}\left[\begin{array}{c}
\mathbf{0} \\
\mathbf{G}_{i}
\end{array}\right] \int_{t-\tau(t)}^{t} \dot{\mathbf{x}}(\varphi) d \varphi \\
& =\sum_{i=1}^{p} w_{i}\left[\begin{array}{l}
\mathbf{x}(t) \\
\mathbf{u}(t)
\end{array}\right]^{\mathrm{T}}\left(\mathbf{P}^{\mathrm{T}}\left[\begin{array}{cc}
\mathbf{A}_{i} & \mathbf{B}_{i} \\
\mathbf{G}_{i} & -\mathbf{I}
\end{array}\right]+\left[\begin{array}{cc}
\mathbf{A}_{i} & \mathbf{B}_{i} \\
\mathbf{G}_{i} & -\mathbf{I}
\end{array}\right]^{\mathrm{T}} \mathbf{P}\right)\left[\begin{array}{l}
\mathbf{x}(t) \\
\mathbf{u}(t)
\end{array}\right] \\
& -\int_{t-\tau(t)}^{t} 2 \sum_{i=1}^{p} w_{i}\left[\begin{array}{l}
\mathbf{x}(t) \\
\mathbf{u}(t)
\end{array}\right]^{\mathrm{T}} \mathbf{P}^{\mathrm{T}}\left[\begin{array}{c}
\mathbf{0} \\
\mathbf{G}_{i}
\end{array}\right] \dot{\mathbf{x}}(\varphi) d \varphi
\end{aligned}
$$

where $\mathbf{P}=\left[\begin{array}{ll}\mathbf{P}_{1} & \mathbf{0} \\ \mathbf{P}_{2} & \mathbf{P}_{3}\end{array}\right] \in \mathfrak{R}^{(n+m) \times(n+m)}, \mathbf{P}_{2} \in \mathfrak{R}^{m \times n}$ and $\mathbf{P}_{3} \in \mathfrak{R}^{m \times m}$. From [15], we have the following inequality.

$$
-2 \mathbf{a}(t)^{\mathrm{T}} \mathbf{N} \dot{\mathbf{x}}(\varphi) \leq\left[\begin{array}{c}
\mathbf{a}(t) \\
\dot{\mathbf{x}}(\varphi)
\end{array}\right]^{\mathrm{T}}\left[\begin{array}{cc}
\hat{\mathbf{R}} & \mathbf{Y}-\mathbf{N} \\
\mathbf{Y}^{\mathrm{T}}-\mathbf{N}^{\mathrm{T}} & \mathbf{R}
\end{array}\right]\left[\begin{array}{c}
\mathbf{a}(t) \\
\dot{\mathbf{x}}(\varphi)
\end{array}\right]
$$

where $\left[\begin{array}{cc}\hat{\mathbf{R}} & \mathbf{Y} \\ \mathbf{Y}^{\mathrm{T}} & \mathbf{R}\end{array}\right] \geq 0$. Let $\mathbf{a}(t)=\left[\begin{array}{l}\mathbf{x}(t) \\ \mathbf{u}(t)\end{array}\right], \mathbf{N}=\mathbf{Y}=\sum_{i=1}^{p} w_{i} \mathbf{Y}_{i}, \quad \mathbf{Y}_{i}=\mathbf{P}^{\mathrm{T}}\left[\begin{array}{c}\mathbf{0} \\ \mathbf{G}_{i}\end{array}\right], \hat{\mathbf{R}}=\sum_{i=1}^{p} w_{i} \hat{\mathbf{R}}_{i}$, $\hat{\mathbf{R}}_{i}=\hat{\mathbf{R}}_{i}^{\mathrm{T}} \in \mathfrak{R}^{(n+m) \times(n+m)}, i=1,2, \ldots, p$. From (14), we have,

$$
\begin{aligned}
& -2 \sum_{i=1}^{p} w_{i}\left[\begin{array}{c}
\mathbf{x}(t) \\
\mathbf{u}(t)
\end{array}\right]^{\mathrm{T}} \mathbf{P}^{\mathrm{T}}\left[\begin{array}{c}
\mathbf{0} \\
\mathbf{G}_{i}
\end{array}\right] \dot{\mathbf{x}}(\varphi) \leq \sum_{i=1}^{p} w_{i}\left[\begin{array}{c}
\mathbf{x}(t) \\
\mathbf{u}(t)
\end{array}\right]^{\mathrm{T}} \hat{\mathbf{R}}_{i}\left[\begin{array}{l}
\mathbf{x}(t) \\
\mathbf{u}(t)
\end{array}\right]+\dot{\mathbf{x}}(\varphi)^{\mathrm{T}} \mathbf{R} \dot{\mathbf{x}}(\varphi) \\
& \Rightarrow-\int_{t-\tau(t)}^{t} 2 \sum_{i=1}^{p} w_{i}\left[\begin{array}{c}
\mathbf{x}(t) \\
\mathbf{u}(t)
\end{array}\right]^{\mathrm{T}} \mathbf{P}^{\mathrm{T}}\left[\begin{array}{c}
\mathbf{0} \\
\mathbf{G}_{i}
\end{array}\right] \dot{\mathbf{x}}(\varphi) \leq \tau(t) \sum_{i=1}^{p} w_{i}\left[\begin{array}{r}
\mathbf{x}(t) \\
\mathbf{u}(t)
\end{array}\right]^{\mathrm{T}} \hat{\mathbf{R}}_{i}\left[\begin{array}{l}
\mathbf{x}(t) \\
\mathbf{u}(t)
\end{array}\right]+\int_{t-\tau(t)}^{t} \dot{\mathbf{x}}(\varphi)^{\mathrm{T}} \mathbf{R} \dot{\mathbf{x}}(\varphi)
\end{aligned}
$$

From (13), (15), and the fact that $0<\tau(t) \leq h$, 


$$
\begin{aligned}
V_{1}(t) & \leq \sum_{i=1}^{p} w_{i}\left[\begin{array}{c}
\mathbf{x}(t) \\
\mathbf{u}(t)
\end{array}\right]^{\mathrm{T}}\left(\mathbf{P}^{\mathrm{T}}\left[\begin{array}{cc}
\mathbf{A}_{i} & \mathbf{B}_{i} \\
\mathbf{G}_{i} & -\mathbf{I}
\end{array}\right]+\left[\begin{array}{cc}
\mathbf{A}_{i} & \mathbf{B}_{i} \\
\mathbf{G}_{i} & -\mathbf{I}
\end{array}\right]^{\mathrm{T}} \mathbf{P}+h \hat{\mathbf{R}}_{i}\right)\left[\begin{array}{c}
\mathbf{x}(t) \\
\mathbf{u}(t)
\end{array}\right] \\
& +\int_{t-\tau(t)}^{t} \dot{\mathbf{x}}(\varphi)^{\mathrm{T}} \mathbf{R} \dot{\mathbf{x}}(\varphi) d \varphi
\end{aligned}
$$

From (2) and (11),

$$
\begin{aligned}
\dot{V}_{2}(t) & =h \dot{\mathbf{x}}(t)^{\mathrm{T}} \mathbf{R} \dot{\mathbf{x}}(t)-\int_{t-h}^{t} \dot{\mathbf{x}}(\varphi)^{\mathrm{T}} \mathbf{R} \dot{\mathbf{x}}(\varphi) d \varphi \\
& =\sum_{i=1}^{p} \sum_{j=1}^{p} w_{i} w_{j} h\left[\begin{array}{l}
\mathbf{x}(t) \\
\mathbf{u}(t)
\end{array}\right]^{\mathrm{T}}\left[\begin{array}{l}
\mathbf{A}_{i}{ }^{\mathrm{T}} \\
\mathbf{B}_{i}{ }^{\mathrm{T}}
\end{array}\right] \mathbf{R}\left[\begin{array}{l}
\mathbf{A}_{j}{ }^{\mathrm{T}} \\
\mathbf{B}_{j}{ }^{\mathrm{T}}
\end{array}\right]^{\mathrm{T}}\left[\begin{array}{l}
\mathbf{x}(t) \\
\mathbf{u}(t)
\end{array}\right]-\int_{t-h}^{t} \dot{\mathbf{x}}(\varphi)^{\mathrm{T}} \mathbf{R} \dot{\mathbf{x}}(\varphi) d \varphi
\end{aligned}
$$

From (10), (16) and (17), we have,

$$
\dot{V}(t) \leq\left[\begin{array}{l}
\mathbf{x}(t) \\
\mathbf{u}(t)
\end{array}\right]^{\mathrm{T}} \mathbf{Q}\left[\begin{array}{l}
\mathbf{x}(t) \\
\mathbf{u}(t)
\end{array}\right]
$$

where

$$
\begin{gathered}
\mathbf{Q}=\sum_{i=1}^{p} \sum_{j=1}^{p} w_{i} w_{j}\left(\mathbf{P}^{\mathrm{T}}\left[\begin{array}{cc}
\mathbf{A}_{i} & \mathbf{B}_{i} \\
\mathbf{G}_{i} & -\mathbf{I}
\end{array}\right]+\left[\begin{array}{cc}
\mathbf{A}_{i} & \mathbf{B}_{i} \\
\mathbf{G}_{i} & -\mathbf{I}
\end{array}\right]^{\mathrm{T}} \mathbf{P}+h \hat{\mathbf{R}}_{i}+h\left[\begin{array}{l}
\mathbf{A}_{i}^{\mathrm{T}} \\
\mathbf{B}_{i}^{\mathrm{T}}
\end{array}\right] \mathbf{R}\left[\begin{array}{l}
\mathbf{A}_{j}^{\mathrm{T}} \\
\mathbf{B}_{j}^{\mathrm{T}}
\end{array}\right]^{\mathrm{T}}\right) \\
\text { Let } \mathbf{X}=\left[\begin{array}{cc}
\mathbf{X}_{1} & \mathbf{0} \\
\mathbf{X}_{2} & \mathbf{X}_{3}
\end{array}\right]=\mathbf{P}^{-1} \in \mathfrak{R}^{(n+m) \times(n+m)}, \mathbf{X}_{1}=\mathbf{X}_{1}^{\mathrm{T}} \in \mathfrak{R}^{n \times n}, \mathbf{X}_{2} \in \mathfrak{R}^{m \times n}, \mathbf{X}_{3} \in \mathfrak{R}^{m \times m}
\end{gathered}
$$

From (18),

$$
\dot{V}(t) \leq\left[\begin{array}{l}
\mathbf{x}(t) \\
\mathbf{u}(t)
\end{array}\right]^{\mathrm{T}} \mathbf{X}^{-1^{\mathrm{T}}} \mathbf{X}^{\mathrm{T}} \mathbf{Q} \mathbf{X} \mathbf{X}^{-1}\left[\begin{array}{l}
\mathbf{x}(t) \\
\mathbf{u}(t)
\end{array}\right]
$$

It can be seen that $\dot{V}(t) \leq 0$ when $\mathbf{X}^{\mathrm{T}} \mathbf{Q} \mathbf{X}<0$ which implies the asymptotic stability of the fuzzy control system. Let $\mathbf{M}=\mathbf{M}^{\mathrm{T}}=\mathbf{R}^{-1}$, 


$$
\begin{aligned}
& \mathbf{M}_{i}=\mathbf{M}_{i}{ }^{\mathrm{T}}=\mathbf{X}^{\mathrm{T}} \hat{\mathbf{R}}_{i} \mathbf{X}=\left[\begin{array}{cc}
\mathbf{M}_{i}^{(11)} & \mathbf{M}_{i}^{(21)^{\mathrm{T}}} \\
\mathbf{M}_{i}^{(21)} & \mathbf{M}_{i}^{(22)}
\end{array}\right] \in \mathfrak{R}^{(n+m) \times(n+m)}, \mathbf{M}_{i}^{(11)}=\mathbf{M}_{i}^{(11)^{\mathrm{T}}} \in \mathfrak{R}^{n \times n}, \mathbf{M}_{i}^{(21)} \in \mathfrak{R}^{m \times n}, \\
& \mathbf{M}_{i}^{(22)}=\mathbf{M}_{i}^{(22)^{\mathrm{T}}} \in \mathfrak{R}^{m \times m}, \mathbf{G}_{i}=\mathbf{N}_{i} \mathbf{X}_{1}^{-1} \text { and } \mathbf{N}_{i} \in \mathfrak{R}^{m \times n} \text { for } i=1,2, \ldots, p \text {. }
\end{aligned}
$$

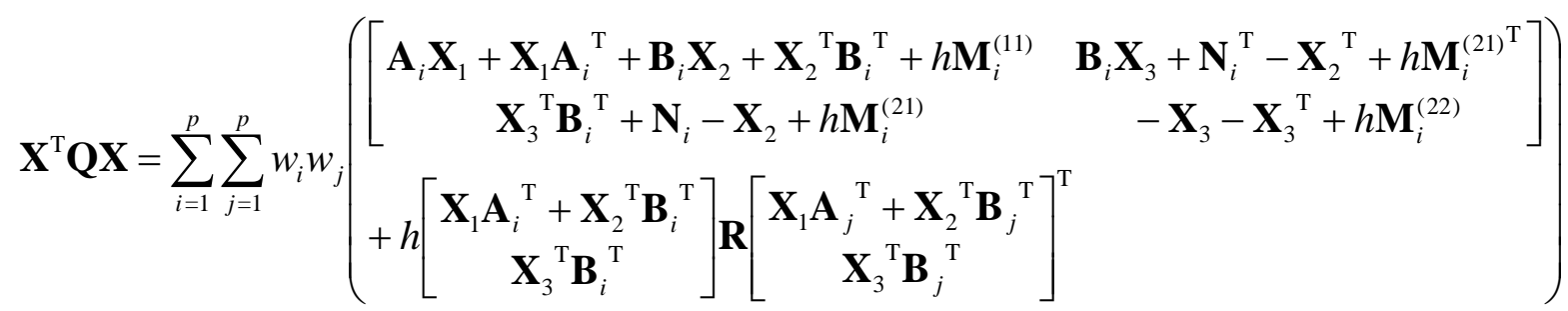

By Schur complement, $\mathbf{X}^{\mathrm{T}} \mathbf{Q X}<0$ is equivalent to the following inequality.

$\sum_{j=1}^{p} w_{i} \overline{\mathbf{Q}}_{i}<0$

where

$$
\overline{\mathbf{Q}}_{i}=\left[\begin{array}{ccc}
\mathbf{A}_{i} \mathbf{X}_{1}+\mathbf{X}_{1} \mathbf{A}_{i}^{\mathrm{T}}+\mathbf{B}_{i} \mathbf{X}_{2}+\mathbf{X}_{2}{ }^{\mathrm{T}} \mathbf{B}_{i}{ }^{\mathrm{T}}+h \mathbf{M}_{i}^{(11)} & \mathbf{B}_{i} \mathbf{X}_{3}+\mathbf{N}_{i}{ }^{\mathrm{T}}-\mathbf{X}_{2}{ }^{\mathrm{T}}+h \mathbf{M}_{i}^{(21){ }^{\mathrm{T}}} & h \mathbf{X}_{1} \mathbf{A}_{i}{ }^{\mathrm{T}}+h \mathbf{X}_{2}{ }^{\mathrm{T}} \mathbf{B}_{i}^{\mathrm{T}} \\
\mathbf{X}_{3}{ }^{\mathrm{T}} \mathbf{B}_{i}{ }^{\mathrm{T}}+\mathbf{N}_{i}-\mathbf{X}_{2}+h \mathbf{M}_{i}{ }^{(21)} & -\mathbf{X}_{3}-\mathbf{X}_{3}{ }^{\mathrm{T}}+h \mathbf{M}_{i}^{(22)} & h \mathbf{X}_{3}^{\mathrm{T}} \mathbf{B}_{i}{ }^{\mathrm{T}} \\
h \mathbf{A}_{i} \mathbf{X}_{1}+h \mathbf{B}_{i} \mathbf{X}_{2} & h \mathbf{B}_{i} \mathbf{X}_{3} & -h \mathbf{M}
\end{array}\right]
$$

$, i=1,2, \ldots, p$. It can be seen that (22) holds when $\overline{\mathbf{Q}}_{i}<0, i=1,2, \ldots, p$. Furthermore,

the inequality of (14) holds when $\left[\begin{array}{cc}\hat{\mathbf{R}} & \mathbf{Y} \\ \mathbf{Y}^{\mathrm{T}} & \mathbf{R}\end{array}\right] \geq 0=\sum_{i=1}^{p} w_{i}\left[\begin{array}{cc}\hat{\mathbf{R}}_{i} & \mathbf{Y}_{i} \\ \mathbf{Y}_{i}^{\mathrm{T}} & \mathbf{R}\end{array}\right] \geq 0$. Considering,

$$
\left[\begin{array}{cc}
\mathbf{X} & \mathbf{0} \\
\mathbf{0} & \mathbf{X}_{1}
\end{array}\right]^{\mathrm{T}}\left[\begin{array}{cc}
\hat{\mathbf{R}}_{i} & \mathbf{Y}_{i} \\
\mathbf{Y}_{i}^{\mathrm{T}} & \mathbf{R}
\end{array}\right]\left[\begin{array}{cc}
\mathbf{X} & \mathbf{0} \\
\mathbf{0} & \mathbf{X}_{1}
\end{array}\right] \geq 0=\left[\begin{array}{cc}
\mathbf{X}^{\mathrm{T}} \hat{\mathbf{R}}_{i} \mathbf{X} & \mathbf{X}^{\mathrm{T}} \mathbf{Y}_{i} \mathbf{X}_{1} \\
\mathbf{X}_{1} \mathbf{Y}_{i}^{\mathrm{T}} \mathbf{X} & \mathbf{X M}^{-1} \mathbf{X}
\end{array}\right] \geq 0, i=1,2, \ldots, p
$$

It should be noted that the inequalities of (23) are not LMI conditions due to the existence of the term $\mathbf{X} \mathbf{M}^{-1} \mathbf{X}$. With the property that $\mathbf{M}=\mathbf{M}^{\mathrm{T}}>0$, we consider the following inequality,

$$
\left(\mathbf{X}_{1}-\zeta \mathbf{M}\right)^{\mathrm{T}} \mathbf{M}^{-1}\left(\mathbf{X}_{1}-\zeta \mathbf{M}\right)=\mathbf{X}_{1}^{\mathrm{T}} \mathbf{M}^{-1} \mathbf{X}_{1}-\zeta \mathbf{X}_{1}^{\mathrm{T}}-\zeta \mathbf{X}_{1}+\zeta^{2} \mathbf{M}>0
$$


$\Rightarrow \mathbf{X}_{1} \mathbf{M}^{-1} \mathbf{X}_{1}>2 \zeta \mathbf{X}_{1}-\zeta^{2} \mathbf{M}$

where $\zeta$ is a non-zero positive scalar. From (24), the holding of the following LMIs implies the holding of (23)

$$
\left[\begin{array}{cc}
\mathbf{X}^{\mathrm{T}} \mathbf{R}_{i} \mathbf{X} & \mathbf{X}^{\mathrm{T}} \mathbf{Y}_{i} \mathbf{X}_{1} \\
\mathbf{X}_{1} \mathbf{Y}_{i}^{\mathrm{T}} \mathbf{X} & 2 \zeta \mathbf{X}_{1}-\zeta^{2} \mathbf{M}
\end{array}\right] \geq 0=\left[\begin{array}{ccc}
\mathbf{M}_{i}^{(11)} & \mathbf{M}_{i}^{(21)^{\mathrm{T}}} & \mathbf{0} \\
\mathbf{M}_{i}^{(21)} & \mathbf{M}_{i}^{(22)} & \mathbf{N}_{i} \\
\mathbf{0} & \mathbf{N}_{i}{ }^{\mathrm{T}} & 2 \zeta \mathbf{X}_{1}-\zeta^{2} \mathbf{M}
\end{array}\right] \geq 0, i=1,2, \ldots, p
$$

It can be seen that the fuzzy control system is asymptotically stable if the stability conditions of (22) and (25) are satisfied.

\section{B. Performance Design}

The LMI-based performance conditions are derived for the fuzzy control system. The system performance is quantitatively measured by the following performance index which is commonly used in the optimal control techniques [16].

$$
J=\int_{0}^{\infty}\left[\begin{array}{l}
\mathbf{x}(t) \\
\mathbf{u}(t)
\end{array}\right]^{\mathrm{T}}\left[\begin{array}{cc}
\mathbf{J}_{1} & \mathbf{J}_{2} \\
\mathbf{J}_{2} & \mathbf{J}_{3}
\end{array}\right]\left[\begin{array}{l}
\mathbf{x}(t) \\
\mathbf{u}(t)
\end{array}\right] d t
$$

where $\quad \mathbf{J}_{1}=\mathbf{J}_{1}{ }^{\mathrm{T}} \in \mathfrak{R}^{n \times n}>0 \quad, \quad \mathbf{J}_{2} \in \mathfrak{R}^{n \times m} \quad, \quad \mathbf{J}_{3}=\mathbf{J}_{3}{ }^{\mathrm{T}} \in \mathfrak{R}^{m \times m}>0 \quad$ and

$$
\begin{aligned}
& {\left[\begin{array}{cc}
\mathbf{J}_{1} & \mathbf{J}_{2} \\
\mathbf{J}_{2}{ }^{\mathrm{T}} & \mathbf{J}_{3}
\end{array}\right] \in \mathfrak{R}^{(n+m) \times(n+m)}>0 \text {. From (6) and (26), we have, }} \\
& J=\int_{0}^{\infty}\left[\mathbf{x}(t)^{\mathrm{T}}\left(\sum_{i=1}^{p} w_{i} \mathbf{G}_{i} \mathbf{x}(t-\tau(t))\right)^{\mathrm{T}}\left[\begin{array}{cc}
\mathbf{J}_{1} & \mathbf{J}_{2} \\
\mathbf{J}_{2}{ }^{\mathrm{T}} & \mathbf{J}_{3}
\end{array}\right]\left[\begin{array}{c}
\mathbf{x}(t) \\
\sum_{j=1}^{p} w_{j} \mathbf{G}_{j} \mathbf{x}(t-\tau(t))
\end{array}\right] d t\right. \\
& =\int_{0}^{\infty}\left[\begin{array}{c}
\mathbf{x}(t) \\
\mathbf{x}(t-\tau(t))
\end{array}\right]^{\mathrm{T}}\left[\begin{array}{cc}
\mathbf{I} & \mathbf{0} \\
\mathbf{0} & \sum_{i=1}^{p} w_{i} \mathbf{G}_{i}{ }^{\mathrm{T}}
\end{array}\right]\left[\begin{array}{cc}
\mathbf{J}_{1} & \mathbf{J}_{2} \\
\mathbf{J}_{2}{ }^{\mathrm{T}} & \mathbf{J}_{3}
\end{array}\right]\left[\begin{array}{cc}
\mathbf{I} & \mathbf{0} \\
\mathbf{0} & \sum_{j=1}^{p} w_{j} \mathbf{G}_{j}
\end{array}\right]\left[\begin{array}{c}
\mathbf{x}(t) \\
\mathbf{x}(t-\tau(t))
\end{array}\right] d t
\end{aligned}
$$


Let $J<\eta \int_{0}^{\infty}\left[\begin{array}{c}\mathbf{x}(t) \\ \mathbf{x}(t-\tau(t))\end{array}\right]^{\mathrm{T}}\left[\begin{array}{cc}\mathbf{X}_{1}^{-1} \mathbf{X}_{1}^{-1} & \mathbf{0} \\ \mathbf{0} & \mathbf{X}_{1}^{-1} \mathbf{X}_{1}^{-1}\end{array}\right]\left[\begin{array}{c}\mathbf{x}(t) \\ \mathbf{x}(t-\tau(t))\end{array}\right] d t$ where $\eta$ is a nonzero positive scalar. Based on this condition and from (27), we have,

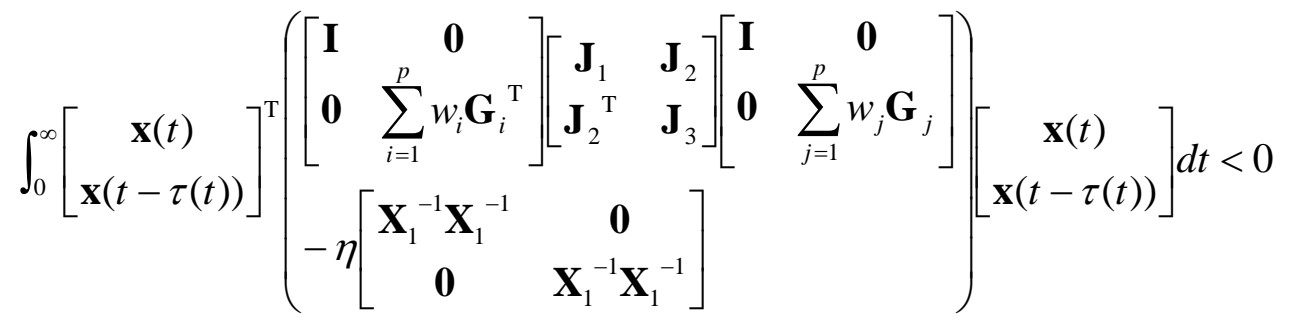

The system performance can be attenuated the performance index $J$ to a to prescribed level of $\eta$. From (28) and let $\mathbf{G}_{i}=\mathbf{N}_{i} \mathbf{X}_{1}^{-1}, i=1,2, \ldots, p$, we have,

$\int_{0}^{\infty}\left[\begin{array}{c}\mathbf{x}(t) \\ \mathbf{x}(t-\tau(t))\end{array}\right]^{\mathrm{T}}\left[\begin{array}{cc}\mathbf{X}_{1}^{-1} & \mathbf{0} \\ \mathbf{0} & \mathbf{X}_{1}^{-1}\end{array}\right] \mathbf{W}\left[\begin{array}{cc}\mathbf{X}_{1}^{-1} & \mathbf{0} \\ \mathbf{0} & \mathbf{X}_{1}^{-1}\end{array}\right]\left[\begin{array}{c}\mathbf{x}(t) \\ \mathbf{x}(t-\tau(t))\end{array}\right] d t<0$

where

$\mathbf{W}=\left[\begin{array}{cc}\mathbf{X}_{1} & \mathbf{0} \\ \mathbf{0} & \sum_{i=1}^{p} w_{i} \mathbf{N}_{i}{ }^{\mathrm{T}}\end{array}\right]\left[\begin{array}{cc}\mathbf{J}_{1} & \mathbf{J}_{2} \\ \mathbf{J}_{2}{ }^{\mathrm{T}} & \mathbf{J}_{3}\end{array}\right]\left[\begin{array}{cc}\mathbf{X}_{1} & \mathbf{0} \\ \mathbf{0} & \sum_{j=1}^{p} w_{j} \mathbf{N}_{j}\end{array}\right]-\eta\left[\begin{array}{ll}\mathbf{I} & \mathbf{0} \\ \mathbf{0} & \mathbf{I}\end{array}\right]$

It can be seen that the inequality of (29) holds when $\mathbf{W}<0$. From (30) and by Schur complement, $\mathbf{W}<0$ is equivalent to the following inequality.

$\sum_{i=1}^{p} w_{i} \mathbf{W}_{i}<0$

where $\left[\begin{array}{cc}\mathbf{K}_{1} & \mathbf{K}_{2} \\ \mathbf{K}_{2}{ }^{\mathrm{T}} & \mathbf{K}_{3}\end{array}\right]=\left[\begin{array}{cc}\mathbf{J}_{1} & \mathbf{J}_{2} \\ \mathbf{J}_{2}{ }^{\mathrm{T}} & \mathbf{J}_{3}\end{array}\right]^{-1}>0, \mathbf{W}_{i}=\left[\begin{array}{cccc}-\eta \mathbf{I} & \mathbf{0} & \mathbf{X}_{1} & \mathbf{0} \\ \mathbf{0} & -\eta \mathbf{I} & \mathbf{0} & \mathbf{N}_{i}{ }^{\mathrm{T}} \\ \mathbf{X}_{1} & \mathbf{0} & -\mathbf{K}_{1} & -\mathbf{K}_{2} \\ \mathbf{0} & \mathbf{N}_{i} & -\mathbf{K}_{2}{ }^{\mathrm{T}} & -\mathbf{K}_{3}\end{array}\right], i=1,2, \ldots, p$. 
It can be seen that the inequality of (31) holds when $\mathbf{W}_{i}<0, i=1,2, \ldots, p$. The LMI conditions of $\mathbf{W}_{i}<0, i=1,2, \ldots, p$ and $\left[\begin{array}{cc}\mathbf{J}_{1} & \mathbf{J}_{2} \\ \mathbf{J}_{2}{ }^{\mathrm{T}} & \mathbf{J}_{3}\end{array}\right]>0$ are the performance conditions. The system stability and performance conditions are summarized in the following theorem.

Theorem 1: The fuzzy control system formed by the nonlinear system in form of (2) and the fuzzy controller of (6) is guaranteed to be asymptotically stable if there exist constant non-zero positive scalars, $h, \zeta$ and $\eta$, and constant matrices, $\mathbf{X}_{1}=\mathbf{X}_{1}^{\mathrm{T}} \in \mathfrak{R}^{n \times n}, \mathbf{X}_{2} \in \mathfrak{R}^{m \times n}$, $\mathbf{X}_{3} \in \mathfrak{R}^{m \times m}, \mathbf{M}=\mathbf{M}^{\mathrm{T}} \in \mathfrak{R}^{n \times n}, \mathbf{M}_{i}^{(11)}=\mathbf{M}_{i}^{(11)^{\mathrm{T}}} \in \mathfrak{R}^{n \times n}, \mathbf{M}_{i}^{(21)} \in \mathfrak{R}^{m \times n}, \mathbf{M}_{i}^{(22)}=\mathbf{M}_{i}^{(22)^{\mathrm{T}}} \in \mathfrak{R}^{m \times m}$, $\mathbf{N}_{j} \in \mathfrak{R}^{m \times n}, \mathbf{J}_{1}=\mathbf{J}_{1}{ }^{\mathrm{T}} \in \mathfrak{R}^{n \times n}, \mathbf{J}_{2} \in \mathfrak{R}^{n \times m}$ and $\mathbf{J}_{3}=\mathbf{J}_{3}{ }^{\mathrm{T}} \in \mathfrak{R}^{m \times m}$ such that the following LMIbased stability and performance conditions hold.

Stability Conditions:

$\mathbf{X}_{1}>0 ; \mathbf{M}>0$;

$$
\begin{aligned}
& {\left[\begin{array}{ccc}
\mathbf{A}_{i} \mathbf{X}_{1}+\mathbf{X}_{1} \mathbf{A}_{i}{ }^{\mathrm{T}}+\mathbf{B}_{i} \mathbf{X}_{2}+\mathbf{X}_{2}{ }^{\mathrm{T}} \mathbf{B}_{i}{ }^{\mathrm{T}}+h \mathbf{M}_{i}^{(11)} & \mathbf{B}_{i} \mathbf{X}_{3}+\mathbf{N}_{i}{ }^{\mathrm{T}}-\mathbf{X}_{2}{ }^{\mathrm{T}}+h \mathbf{M}_{i}^{(21)} & h \mathbf{X}_{1} \mathbf{A}_{i}{ }^{\mathrm{T}}+h \mathbf{X}_{2}{ }^{\mathrm{T}} \mathbf{B}_{i}{ }^{\mathrm{T}} \\
\mathbf{X}_{3}{ }^{\mathrm{T}} \mathbf{B}_{i}{ }^{\mathrm{T}}+\mathbf{N}_{i}-\mathbf{X}_{2}+h \mathbf{M}_{i}^{(21)} & -\mathbf{X}_{3}-\mathbf{X}_{3}{ }^{\mathrm{T}}+h \mathbf{M}_{i}^{(22)} & h \mathbf{X}_{3}{ }^{\mathrm{T}} \mathbf{B}_{i}^{\mathrm{T}} \\
h \mathbf{A}_{i} \mathbf{X}_{1}+h \mathbf{B}_{i} \mathbf{X}_{2} & h \mathbf{B}_{i} \mathbf{X}_{3} & -h \mathbf{M}
\end{array}\right]<0} \\
& {\left[\begin{array}{ccc}
\mathbf{M}_{i}^{(11)} & \mathbf{M}_{i}^{(21)^{\mathrm{T}}} & \mathbf{0} \\
\mathbf{M}_{i}^{(21)} & \mathbf{M}_{i}^{(22)} & \mathbf{N}_{i} \\
\mathbf{0} & \mathbf{N}_{i}^{\mathrm{T}} & 2 \zeta \mathbf{X}_{1}-\zeta^{2} \mathbf{M}
\end{array}\right] \geq 0, i=1,2, \ldots, p \text { and the feedback gains are defined } \text { as }} \\
& \mathbf{G}_{i}=\mathbf{N}_{i} \mathbf{X}_{1}^{-1}, i=1,2, \ldots, p .
\end{aligned}
$$

Performance Conditions: 


$$
\left[\begin{array}{ll}
\mathbf{J}_{1} & \mathbf{J}_{2} \\
\mathbf{J}_{2} & \mathbf{J}_{3}
\end{array}\right]=\left[\begin{array}{cc}
\mathbf{K}_{1} & \mathbf{K}_{2} \\
\mathbf{K}_{2}{ }^{\mathrm{T}} & \mathbf{K}_{3}
\end{array}\right]^{-1}>0, \mathbf{W}_{i}=\left[\begin{array}{cccc}
-\eta \mathbf{I} & \mathbf{0} & \mathbf{X}_{1} & \mathbf{0} \\
\mathbf{0} & -\eta \mathbf{I} & \mathbf{0} & \mathbf{N}_{i}^{\mathrm{T}} \\
\mathbf{X}_{1} & \mathbf{0} & -\mathbf{K}_{1} & -\mathbf{K}_{2} \\
\mathbf{0} & \mathbf{N}_{i} & -\mathbf{K}_{2}{ }^{\mathrm{T}} & -\mathbf{K}_{3}
\end{array}\right]<0, i=1,2, \ldots, p .
$$

Remark 1: It can be seen that the solution to the stability conditions in Theorem 1 implies $\mathbf{X}_{1}=\mathbf{X}_{1}^{\mathrm{T}}>0$ and $-\mathbf{X}_{3}-\mathbf{X}_{3}{ }^{\mathrm{T}}<0$. As $\mathbf{X}_{1}=\mathbf{X}_{1}{ }^{\mathrm{T}}>0$ and $-\mathbf{X}_{3}-\mathbf{X}_{3}{ }^{\mathrm{T}}<0$, $\mathbf{X}=\left[\begin{array}{cc}\mathbf{X}_{1} & \mathbf{0} \\ \mathbf{X}_{2} & \mathbf{X}_{3}\end{array}\right]$ is a non-singular matrix. Hence, there must exist $\mathbf{P}^{-1}=\mathbf{X}$ if the stability conditions in Theorem 1 has solution.

IV. Application ExAmple

An application example on stabilizing a cart-pole typed inverted pendulum [17] is given in this section.

Step I) The dynamic equations of the inverted pendulum on the cart [17] is given by,

$$
\dot{x}_{1}(t)=x_{2}(t)
$$

$\dot{x}_{2}(t)=\frac{\left(\begin{array}{l}-F_{1}(M+m) x_{2}(t)-m^{2} I^{2} x_{2}(t)^{2} \sin x_{1}(t) \cos x_{1}(t)+F_{0} m l x_{4}(t) \cos x_{1}(t) \\ +(M+m) m g l \sin x_{1}(t)-m l \cos x_{1}(t) u(t)\end{array}\right)}{(M+m)\left(J+m l^{2}\right)-m^{2} l^{2}\left(\cos x_{1}(t)\right)^{2}}$

$$
\dot{x}_{3}(t)=x_{4}(t)
$$

$$
\dot{x}_{4}(t)=\frac{\left(\begin{array}{l}
F_{1} m l x_{2}(t) \cos x_{1}(t)+\left(J+m l^{2}\right) m l x_{2}(t)^{2} \sin x_{1}(t)-F_{0}\left(J+m l^{2}\right) x_{4}(t) \\
-m^{2} g l^{2} \sin x_{1}(t) \cos x_{1}(t)+\left(J+m l^{2}\right) u(t)
\end{array}\right)}{(M+m)\left(J+m l^{2}\right)-m^{2} l^{2}\left(\cos x_{1}(t)\right)^{2}}
$$


where $x_{1}(t)$ and $x_{2}(t)$ denote the angular displacement (rad) and the angular velocity $(\mathrm{rad} / \mathrm{s})$ of the pendulum from vertical respectively, $x_{3}(t)$ and $x_{4}(t)$ denote the displacement (m) and the velocity (m/s) of the cart respectively, $g=9.8 \mathrm{~m} / \mathrm{s}^{2}$ is the acceleration due to gravity, $m=0.22 \mathrm{~kg}$ is the mass of the pendulum, $M=1.3282 \mathrm{~kg}$ is the mass of the cart, $l$ $=0.304 \mathrm{~m}$ is the length from the center of mass of the pendulum to the shaft axis, $J=$ $\mathrm{ml}^{2} / 3 \mathrm{kgm}^{2}$ is the moment of inertia of the pendulum round the center of mass, $F_{0}=$ $22.915 \mathrm{~N} / \mathrm{m} / \mathrm{s}$ and $F_{1}=0.007056 \mathrm{~N} / \mathrm{rad} / \mathrm{s}$ are the friction factors of the cart and the pendulum respectively, and $u(\mathrm{t})$ is the force $(\mathrm{N})$ applied to the cart. The objective of this application example is to design the proposed fuzzy controller to close the feedback loop such that $x_{1}(t)=x_{3}(t)=0$ at the steady state. The nonlinear plant can be represented by a fuzzy model with two fuzzy rules [17]. The $i$-th rule is given by,

Rule $i$ : IF $x_{1}(t)$ is $\mathrm{M}_{1}^{i}$ THEN $\dot{\mathbf{x}}(t)=\mathbf{A}_{i} \mathbf{x}(t)+\mathbf{B}_{i} u(t)$ for $i=1,2$

The system dynamics are described by,

$\dot{\mathbf{x}}(t)=\sum_{i=1}^{2} w_{i}\left(x_{1}\right)\left(\mathbf{A}_{i} \mathbf{x}(t)+\mathbf{B}_{i} u(t)\right)$

where

$$
\mathbf{x}(t)=\left[x_{1}(t) \quad x_{2}(t) \quad x_{3}(t) \quad x_{4}(t)\right]^{\mathrm{T}}
$$

$$
\begin{aligned}
& \mathbf{A}_{1}=\left[\begin{array}{cccc}
0 & 1 & 0 & 0 \\
(M+m) m g l / a_{1} & -F_{1}(M+m) / a_{1} & 0 & F_{0} m l / a_{1} \\
0 & 0 & 1 & 0 \\
-m^{2} g l^{2} / a_{1} & F_{1} m l / a_{1} & 0 & -F_{0}\left(J+m l^{2}\right) / a_{1}
\end{array}\right], \quad \mathbf{B}_{1}=\left[\begin{array}{c}
0 \\
-m l / a_{1} \\
0 \\
\left(J+m l^{2}\right) / a_{1}
\end{array}\right] ; \\
& \mathbf{A}_{2}=\left[\begin{array}{cccc}
0 & 1 & 0 & 0 \\
\frac{3 \sqrt{3}}{2 \pi}(M+m) m g l / a_{2} & -F_{1}(M+m) / a_{2} & 0 & F_{0} m l \cos (\pi / 3) / a_{2} \\
0 & 0 & 1 & 0 \\
-\frac{3 \sqrt{3}}{2 \pi} m^{2} g l^{2} \cos (\pi / 3) / a_{2} & F_{1} m l \cos (\pi / 3) / a_{2} & 0 & -F_{0}\left(J+m l^{2}\right) / a_{1}
\end{array}\right],
\end{aligned}
$$




$$
\begin{aligned}
& \mathbf{B}_{2}=\left[\begin{array}{c}
0 \\
-m l \cos (\pi / 3) / a_{2} \\
0 \\
\left(J+m l^{2}\right) / a_{2}
\end{array}\right] ; \quad a_{1}=(M+m)\left(J+m l^{2}\right)-m^{2} l^{2} \\
& a_{2}=(M+m)\left(J+m l^{2}\right)-m^{2} l^{2} \cos (\pi / 3)^{2} ; \quad w_{i}\left(x_{1}\right)=\frac{\mu_{\mathrm{M}_{1}^{i}}\left(x_{1}(t)\right)}{\sum_{l=1}^{2} \mu_{\mathrm{M}_{1}^{\prime}}\left(x_{1}(t)\right)} . \quad \text { The membership }
\end{aligned}
$$

functions $\quad$ are $\quad$ defined $\quad$ as $\quad \mu_{\mathrm{M}_{1}^{1}}\left(x_{1}(t)\right)=\left(1-\frac{1}{1+e^{-7\left(x_{1}(t)-\pi / 6\right)}}\right) \frac{1}{1+e^{-7\left(x_{1}(t)+\pi / 6\right)}} \quad$ and $\mu_{\mathrm{M}_{1}^{2}}\left(x_{1}(t)\right)=1-\mu_{\mathrm{M}_{1}^{1}}\left(x_{1}(t)\right)$.

Step II) A fuzzy controller with four sampled-data sub-controller is employed to handle the inverted pendulum. The fuzzy controller is given by,

$$
u(t)=\sum_{j=1}^{2} w_{j}(\mathbf{x}(t)) \mathbf{G}_{j} \mathbf{x}\left(t_{\gamma}\right), t_{\gamma}<t \leq t_{\gamma+1}
$$

Step III) Based on Theorem 1, with $h=0.02 \mathrm{~s}, \zeta=2, \eta=10^{-5}, \mathbf{J}_{1}=\left[\begin{array}{cccc}1 & 0 & 0 & 0 \\ 0 & 1 & 0 & 0 \\ 0 & 0 & 1 & 0 \\ 0 & 0 & 0 & 1\end{array}\right]$, $\mathbf{J}_{2}=\left[\begin{array}{l}0 \\ 0 \\ 0 \\ 0\end{array}\right]$ and $\mathbf{J}_{3}=1$, we have $\mathbf{G}_{1}=\left[\begin{array}{llll}243.9049 & 25.1386 & 0.3358 & 31.0842\end{array}\right]$ and $\mathbf{G}_{2}=$ [691.2348 52.0482 0.7582 42.4703]. To show the effect of the LMI performance 
conditions, another set of feedback gains is obtained with $\mathbf{J}_{1}=\left[\begin{array}{cccc}1 & 0 & 0 & 0 \\ 0 & 1 & 0 & 0 \\ 0 & 0 & 100 & 0 \\ 0 & 0 & 0 & 1\end{array}\right]$,

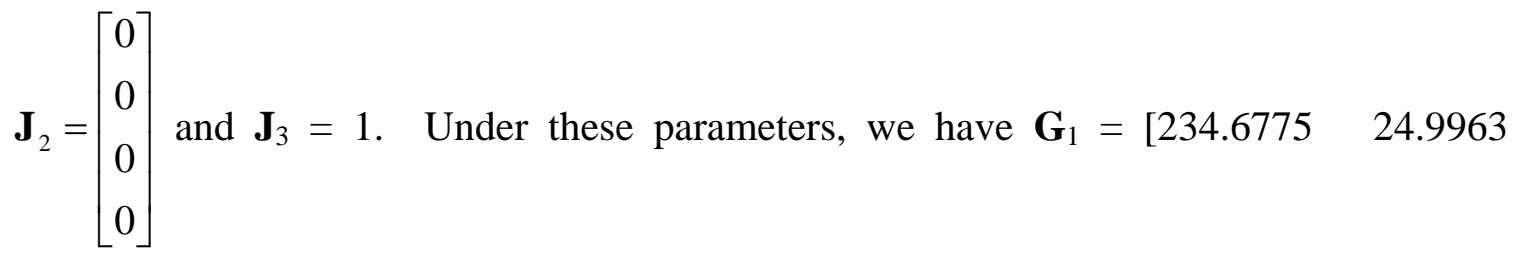

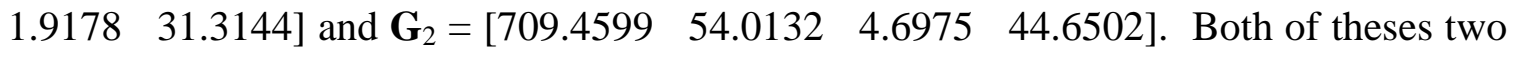
controllers are employed to handle the inverted pendulum.

Fig. 1 shows the system state responses and control signals of the fuzzy control system. It can be seen that both fuzzy controllers with different feedback gains can stabilize the nonlinear plant. The fuzzy controller with $\mathbf{J}_{1}=\left[\begin{array}{cccc}1 & 0 & 0 & 0 \\ 0 & 1 & 0 & 0 \\ 0 & 0 & 100 & 0 \\ 0 & 0 & 0 & 1\end{array}\right]$, which places heavier weight on $x_{3}(t)$, offers better system responses in terms of faster settling time for $x_{3}(t)$. Referring to this figure, it can be seen that the control signals are continuous during the sampling period due to the grade of the membership is continuous changing and there is a jump at the sampling instant in which the control signals of the sampled-data linear sub-controllers change. In this example, the proposed LMI-based stability and performance conditions offer a systematic tool to help achieve a stable and well-performed fuzzy controller with sampled-data sub-control rules.

\section{CONCLUSION}


The stability of the fuzzy control system formed by a nonlinear plant and a fuzzy controller with sampled-data sub-control rules has been investigated. Stability conditions in terms of linear matrix inequalities have been derived based on Lyapunov-based approach. LMI-based performance conditions have been derived to guarantee the system performance. With the LMI-based stability and performance conditions, a stable and well-performed fuzzy controller with sampled-data sub-control rules can be designed for a nonlinear system. An application example has been given to illustrate the effectiveness of the proposed approach.

\section{ACKNOWLEDGEMENT}

The work described in this paper was supported by the Division of Engineering, King's College London.

\section{REFERENCES}

1 Takagi T. and Sugeno M.: 'Fuzzy identification of systems and its applications to modeling and control' IEEE Trans. Sys., Man., Cybern., 1985, smc-15, (1), pp. 116-132.

2 Sugeno M. and Kang G.T.: 'Structure identification of fuzzy model', Fuzzy sets and systems, 1988, 28, pp. 15-33.

3 Chen C.L., Chen P.C., Chen C.K.: 'Analysis and design of fuzzy control system', Fuzzy Sets and Systems, 1993, 57, (2), pp. 125-140. 
4 Wang H.O., Tanaka K., and Griffin M.F.: 'An approach to fuzzy control of nonlinear systems: stability and the design issues’ IEEE Trans. Fuzzy Syst., 1996, 4, (1), pp. 14-23.

5 Boyd S., Ghaoui L., Feron E. and Balakrishnan V.: Linear matrix inequalities in system and control theory. SIAM, 1994.

6 Tanaka K., Ikeda T. and Wang H.O.: 'Fuzzy regulator and fuzzy observer: Relaxed stability conditions and LMI-based designs’, IEEE Trans. Fuzzy Syst., 1998, 6, (2), pp. 250-265.

7 Tanaka K., Ikeda T. and Wang H.O., "Fuzzy regulator and fuzzy observer: Relaxed stability conditions and LMI-based designs,” IEEE Trans. Fuzzy Syst., 1998, 6 (2), pp. 250-265.

8 Kim E. and Lee H.: 'New approaches to relaxed quadratic stability conditions of fuzzy control systems’, IEEE Trans. Fuzzy Syst., 2000, 8, (5), pp. 523-534.

9 Liu X. and Zhang Q.: 'New approaches to $H_{\infty}$ controller designs based on fuzzy observers for T-S fuzzy systems via LMI’, Automatica, 2003, 39, (9), pp. 15711582.

10 Teixeira M.C.M., Assunção E. and Avellar R.G., “On relaxed LMI-based designs for fuzzy regulators and fuzzy observers,” IEEE Trans. on Fuzzy Systems, 2003, 11, (5), pp. 613-623.

11 Fang C.H., Liu Y.S., Kau S.W., Hong L. and Lee C.H., “A new LMI-based approach to relaxed quadratic stabilization of T-S fuzzy control systems,” IEEE Trans. on Fuzzy Systems, 2006, 14, (3), pp. 386-397. 
12 Katayama $H$. and Ichikawa A., " $H_{\infty}$ control for sampled-data nonlinear systems described by Takagi-Sugeno fuzzy systems,” Fuzzy Sets and Systems, 2004, 148, (3), pp. 431-452.

13 Fridman E., Seuret A. and Richard J.P., "Robust sampled-data stabilization of linear systems: an output delay approach,” Automatica, 2004, 40, (8), pp.1441-1446.

14 Lam H.K. and Leung F.H.F., "Sampled-data fuzzy controller for time-delay nonlinear system: LMI-based and fuzzy-model-based approaches,” IEEE Trans. Syst., Man and Cybern., Part B: Cybernetics, 2007, 37, (3), pp. 617-629.

15 Moon Y.S., Park P., Kwon W.H. and Lee Y.S., "Delay-dependent robust stabilization of uncertain state-delayed systems,” Int. Journal of Control, 2001, 74, (14), pp.1447-1455.

16 Anderson B.D.O. and Moore J.B., Optimal Control: Linear Quadratic Methods. Prentice Hall, 1990.

17 Ma X.J. and Sun Z.Q.: 'Analysis and design of fuzzy reduced-dimensional observer and fuzzy functional observer', Fuzzy Sets and Systems, 2001, 120, (1), pp. 35-63. 


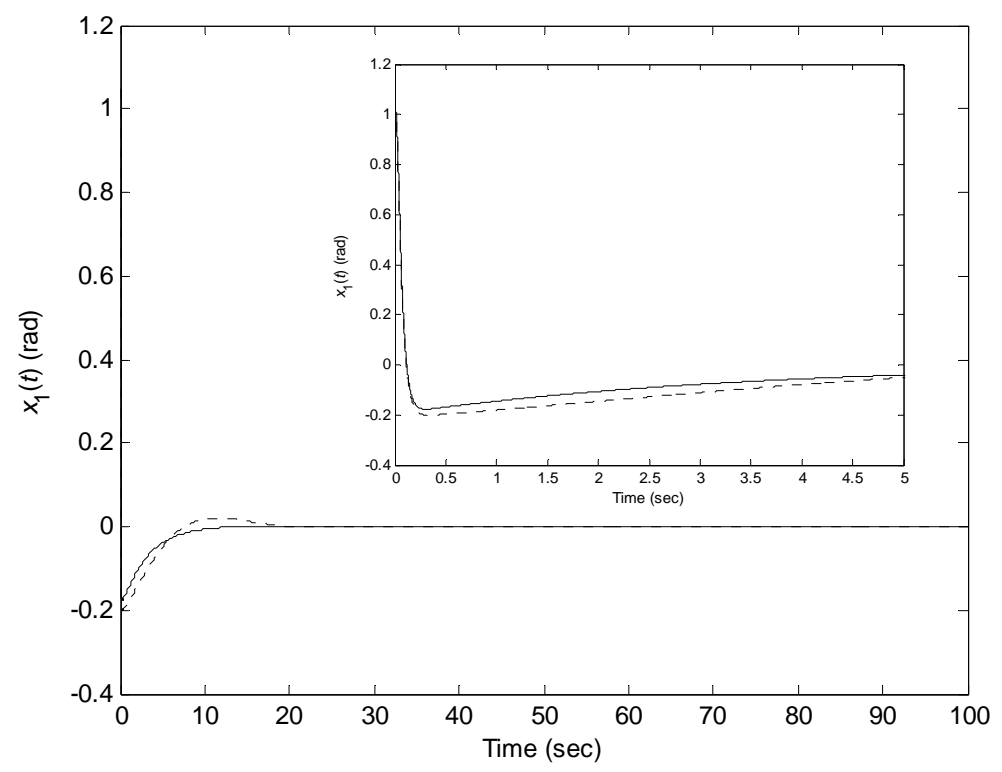

Fig. 1(a). $x_{1}(t)$.

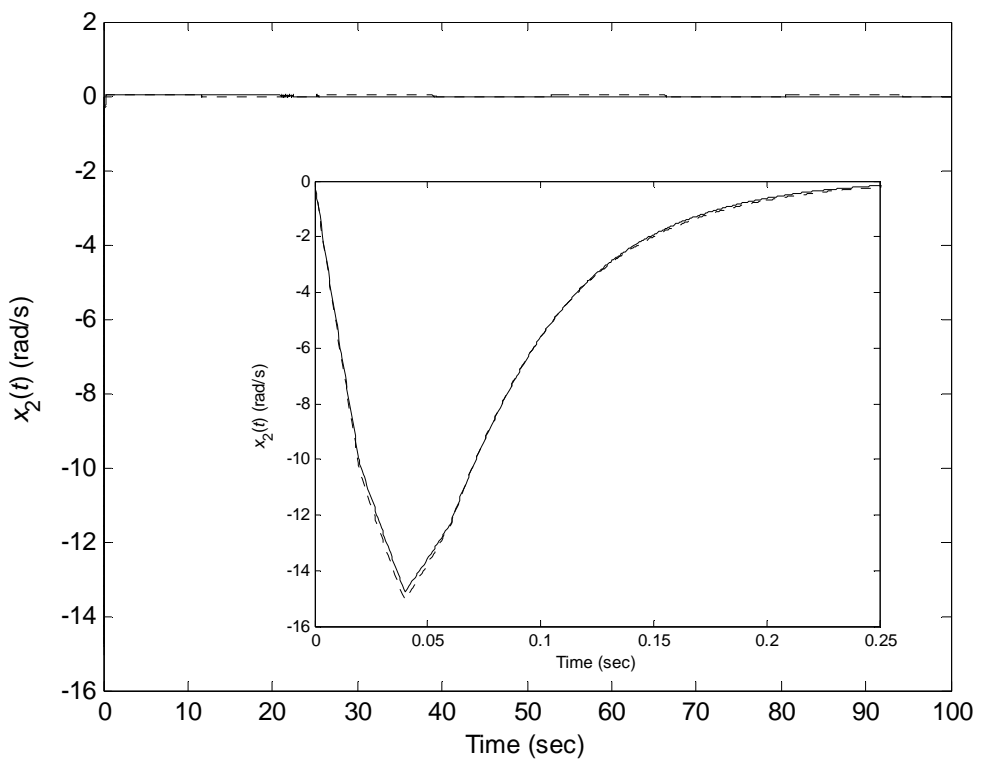

Fig. 1(b). $x_{2}(t)$. 


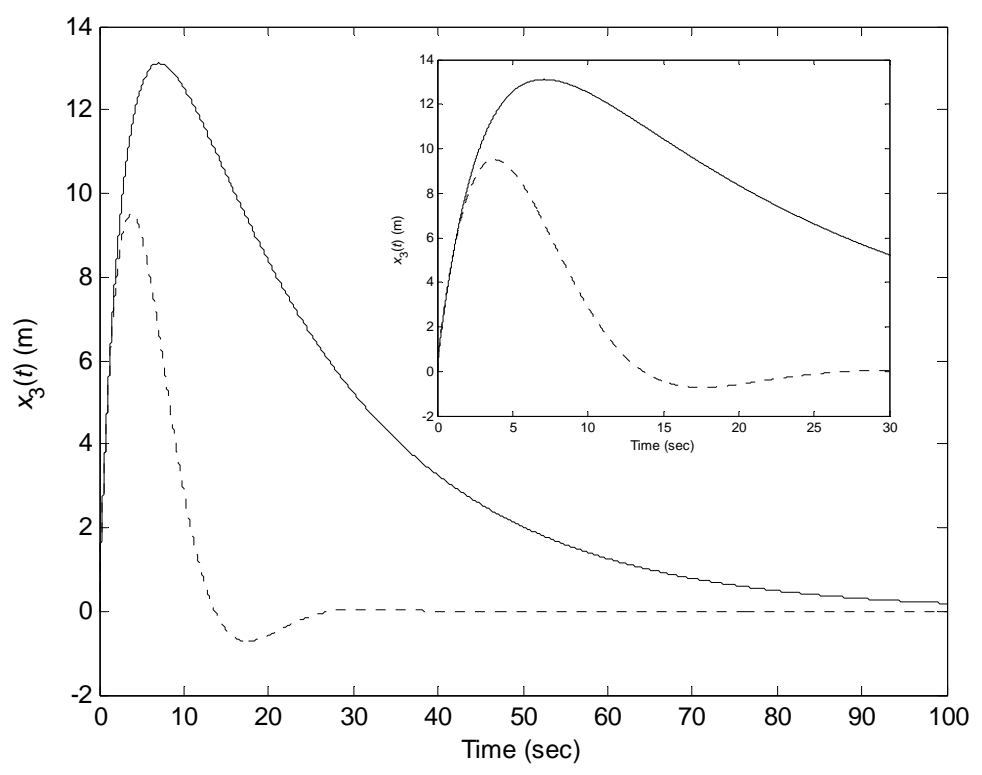

Fig. 1(c). $x_{3}(t)$.

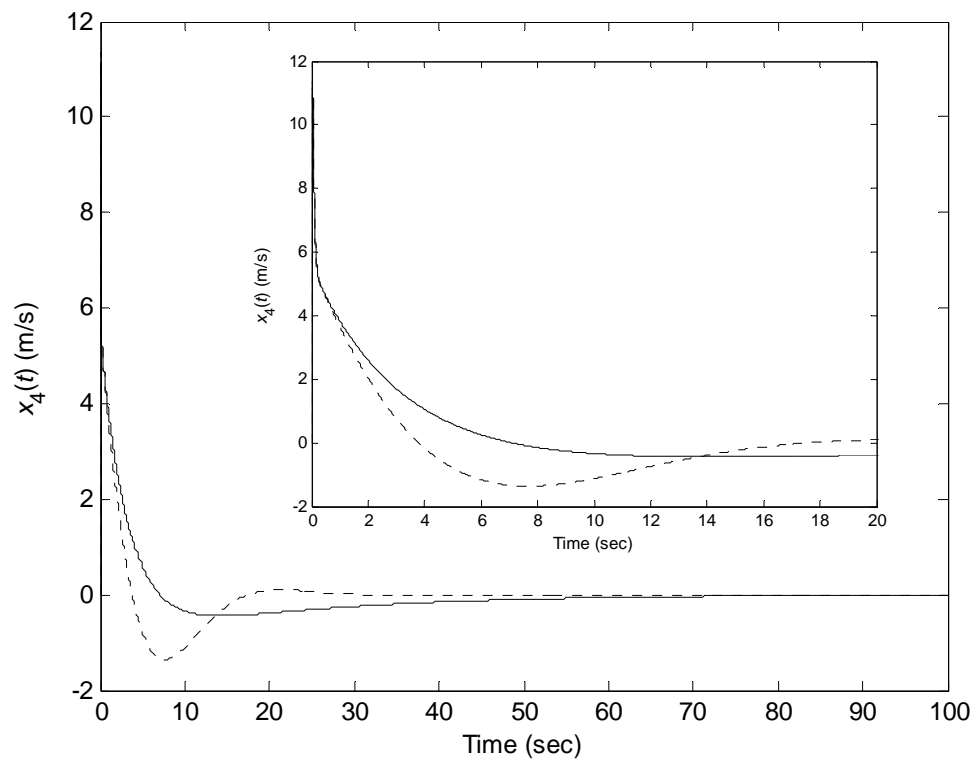

Fig. 1(d). $x_{4}(t)$. 


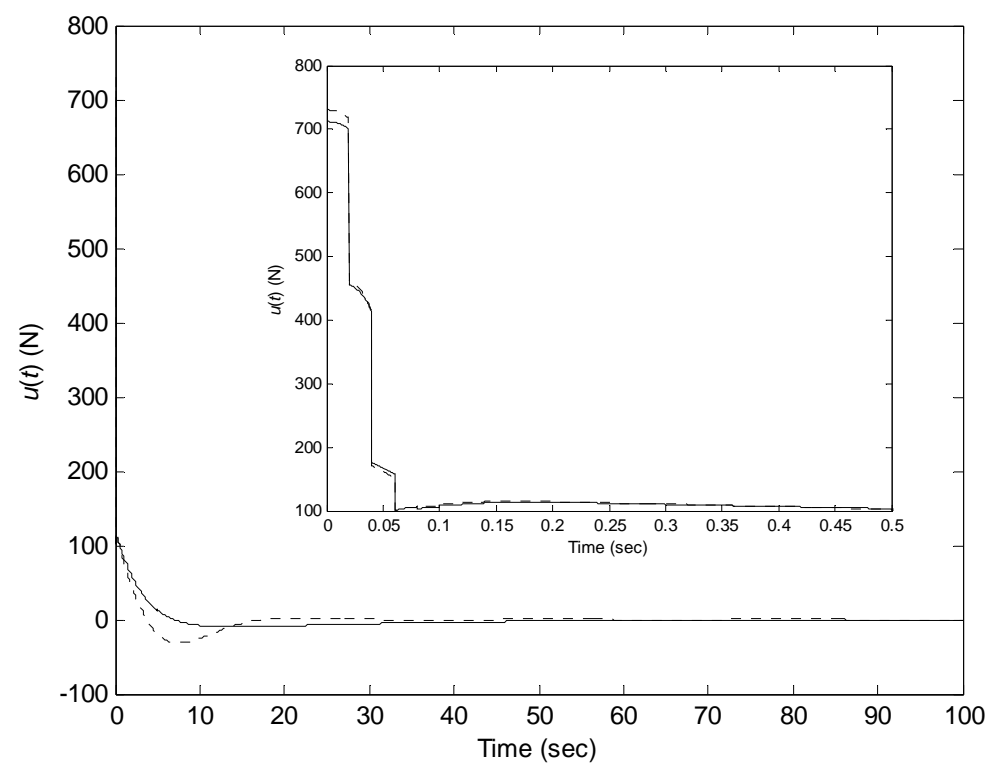

Fig. 1(e). $u(t)$.

Fig. 1. System state responses and control signals of the fuzzy control system with

$$
\mathbf{J}_{1}=\left[\begin{array}{llll}
1 & 0 & 0 & 0 \\
0 & 1 & 0 & 0 \\
0 & 0 & 1 & 0 \\
0 & 0 & 0 & 1
\end{array}\right] \text { (solid lines) and } \mathbf{J}_{1}=\left[\begin{array}{cccc}
1 & 0 & 0 & 0 \\
0 & 1 & 0 & 0 \\
0 & 0 & 100 & 0 \\
0 & 0 & 0 & 1
\end{array}\right] \text { (dotted lines). }
$$

